\title{
FOCAL CEMENTO-OSSEOUS DYSPLASIA OF LOWER JAW IN AN ELDERLY WOMAN: CLINICOPATHOLOGICAL FEATURES AND MANAGEMENT IN A CASE REPORT
}

Swati Kapoor ${ }^{1}$, Gurnam Singh ${ }^{2}$, Dinesh Kumar ${ }^{3}$, Puneet Bajaj ${ }^{4}$, Alka Bhardwaj ${ }^{5}$

${ }^{1}$ Sr. Lecturer, Department of Oral \& Maxillofacial Surgery, Bhojia Dental College \& Hospital, Himachal Pradesh, India

${ }^{2}$ M.D.S., Professor \& HOD, Department of Oral \& Maxillofacial Surgery, Bhojia Dental College \& Hospital, Himachal Pradesh, India ${ }^{3}$ M.D.S., Assistant Professor, Department of Oral \& Maxillofacial Surgery, Bhojia Dental College \& Hospital, Himachal Pradesh, India

${ }^{4}$ M.D.S., Professor \& HOD, Department of Oral Pathology and Microbiology, Bhojia Dental College \& Hospital, Himachal Pradesh, India

${ }^{5}$ Post Graduate Student, Department of Oral Pathology \& Microbiology, Bhojia Dental College \& Hospital, Himachal Pradesh, India

Corresponding Author:

Dinesh Kumar

E-mail

dr_dinesh78@yahoo.com Received: $2^{\text {nd }}$ October 2016 Accepted: $15^{\text {th }}$ November 2016 Online: $20^{\text {th }}$ January 2017

\begin{abstract}
Focal cemento-osseous dysplasia is a benign fibroosseous lesion that can be seen in dentulous and edentulous patients mainly in middle aged women. Although it is asymptomatic condition associated with vital teeth and needs no treatment but symptomatic patients require surgical debridement and sequestrectomy. We present a case report of symptomatic focal cemento-osseous dysplasia which requires surgical intervention.
\end{abstract}

\section{Introduction}

Fibro-osseous lesions are a poorly defined group of lesions affecting the jaws and craniofacial bones. All are characterized by the replacement of bone by cellular fibrous tissue containing foci of mineralization that vary in amount and may be bony or cementum-like in appearance. Many lesions contain an admixture of these calcifications. However, with adequate clinical and radiologic information and an adequate biopsy or surgical specimen, most fibro-osseous lesions of the jaws can be assigned with reasonable certainty to one of the categories as by Waldron :I. Fibrous dysplasia II. Fibro-osseous neoplasms III. Reactive (dysplastic) lesions arising in the tooth-bearing area, although these are presumably of periodontal ligament origin, divided into three types based on their radiologic features; they seem to represent the same pathologic process. a) Periapical cemento -osseous dysplasia b) Focal cementoosseous dysplasia c) Florid cementoosseous dysplasia.

Periapical osseous dysplasia, a subtype of cemento-osseous dysplasia occurs in the anterior mandible. Women over 30 years of age and mandible are predisposed to these lesions and approximately $70 \%$ of the cases are reported in blacks. Periapical cementoosseous dysplasia (PCOD) is invariably an asymptomatic lesion that appears occasionally in radiographs taken for other reasons. Focal cemento-osseous dysplasia (FCOD) and periapical cemento-osseous dysplasia are different names for the same pathological process. Periapical cementoosseous dysplasia is bilateral and is located at the root apices of the mandibular anterior teeth. Microscopic findings of periapical cementoosseous dysplasia are identical to those of Focal 
cemento-osseous dysplasia but later occur in posterior mandible.

Typically, the lesion is asymptomatic and is detected on routine radiographic examination. Radiologically, this lesion has three stages of maturation: pure radiolucent, radiopaque/mixed radiolucent and radiopaque appearances. The early stage of FCOD classically presents with a well defined radiolucency at the apices of mandibular teeth. The radiographic image may be erroneously diagnosed as an endodontic infection. In dentate areas, neither tooth displacement nor root resorption is observed. To assess the lesion, a pulp vitality test can be performed. FCOD lesions at an intermediate stage demonstrate a mixed radiolucent/opaque pattern, with a well-defined radiolucent rim around the radiopacity. At a later stage, these lesions display diffuse radiopacity, often with illdefined borders and a greater proportion of anastomosing, thick curvilinear, poorly cellular bony trabeculae (the so-called'ginger root'pattern). ${ }^{1,2}$

This report describes a case of focal cementoosseous dysplasia.

Keywords: Fibro-osseous lesions, Focal cementoosseous dysplasia, Florid cemento-osseous dysplasia, Mandible

\section{Case report}

A 60 year old woman (Figure 1) reported to the department of oral surgery with a complaint of pain and discomfort at the area of teeth 33,34 since 1 month.
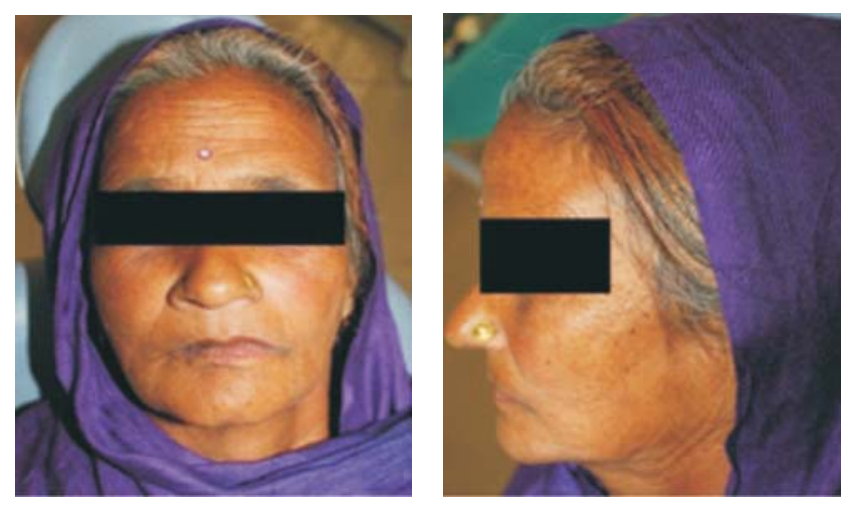

Figure 1: Intraoral radiograph showing normal implant and PLS implant
Extra oral examination shows no obvious swelling or asymmetry. During the intraoral examination; multiple missing teeth, generalised periodontitis, caries and periodontal pocket in region of 33, 34 were found (Figure 2). Lower canine tooth on left side, were found to be tender on percussion.

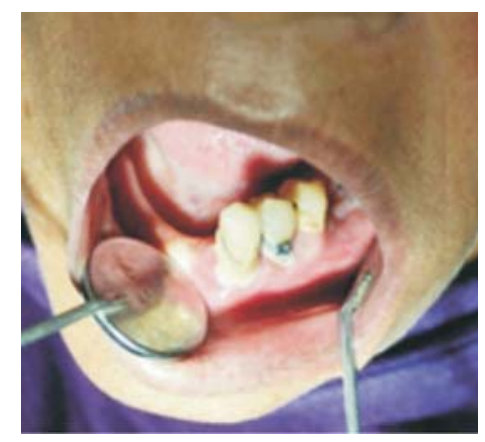

Figure 2: Intraoral view shows multiple missing and carious 33,34 .

To evaluate the reason of pain and discomfort both periapical as well as panoramic radiographs were done. During analysis of both radiographs, it was observed that there was radioopaque lesion with radiolucent margins w.r.t 33 and 34 (Figure 3).

The patient was having no relevant medical history. Routine blood investigation was performed. Total leucocyte count was found to be raised.

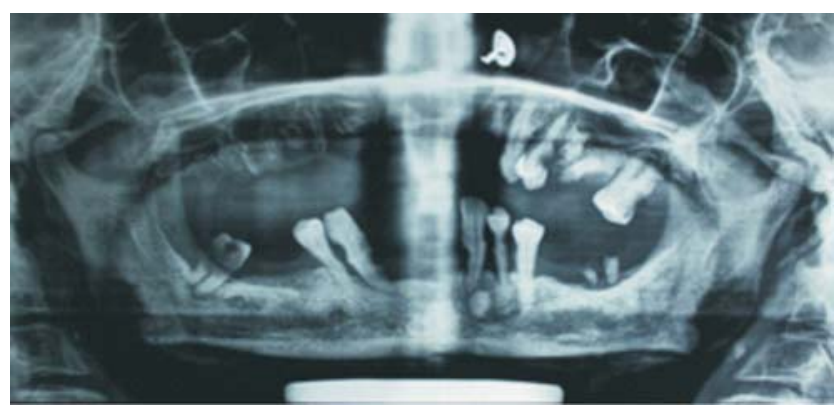

Figure 3: Orthopantomogram shows small well defined radioopaque lesion w.r.t 33,34

Periapical pathoses present wide spectrum of lesions that mimic each other. On the basis of clinical and radiographic findings, a provisional diagnosis of focal cement-osseous dysplasia was made. Other possible differential diagnoses were condensing osteitis, periapical idiopathic osteosclerosis, cemento-ossifying 
fibroma, cementoblastoma, and complex odontoma. Focal cement-osseous dysplasia is characteristically rounded radioopacity with well defined border and is separated from normal bone and root by a thin radiolucent halo. This feature differentiates this lesion from condensing osteitis and idiopathic osteosclerosis. ${ }^{3}$

Under complete sterile and aseptic conditions, profound local anaesthesia was achieved using inferior alveolar and lingual nerve blocks. Triangular flap was raised and lesion was surgically exposed with the help of surgical hand piece and bur along with copious saline irrigation. The tooth was extracted and the periapical lesion was removed (Figure 4).After complete haemostasis, the surgical site was closed with interrupted sutures using 3-0 black silk. The specimen was submitted for histopathological examination. (Figure 5).

The decalcified H\&E stained section shows hard tissue interspersed within the connective tissue stroma. Hard tissue consists of irregular bony trabecular like

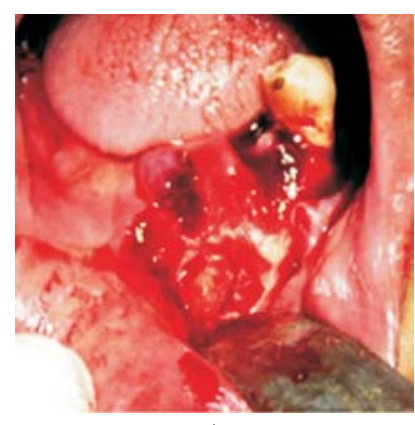

A

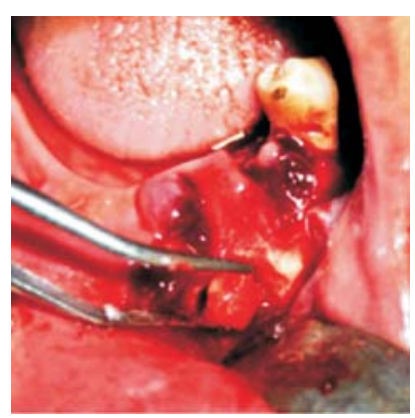

B

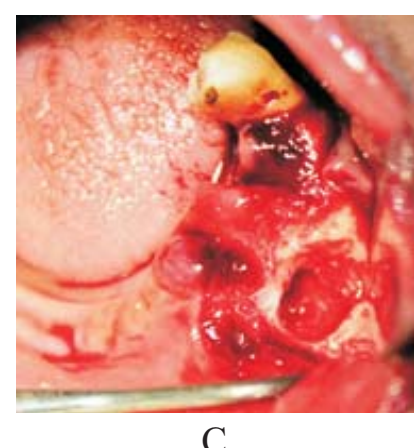

Figure 4: (A) Intraoperative view of the lesion; the mass adjacent to apices of $33 \& 34$ having a slight yellowish colour that can easily be differentiated from bone. (B) Removal of the lesion. (C) Residual cavity after removal of periapical lesion.

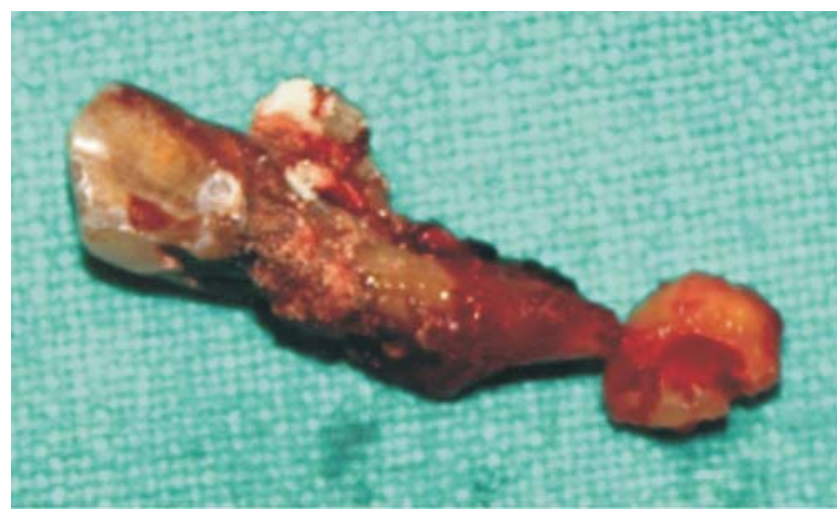

Figure 5: Gross specimen of excised lesion along with involved tooth.

structures with irregular basophilic reversal lines. Small cementicle like structures are also seen. Connective tissue stroma comprises of collagen fibres with plump fibroblasts. All these features suggest the presence of focal cement- osseous lesions (Figure 6).
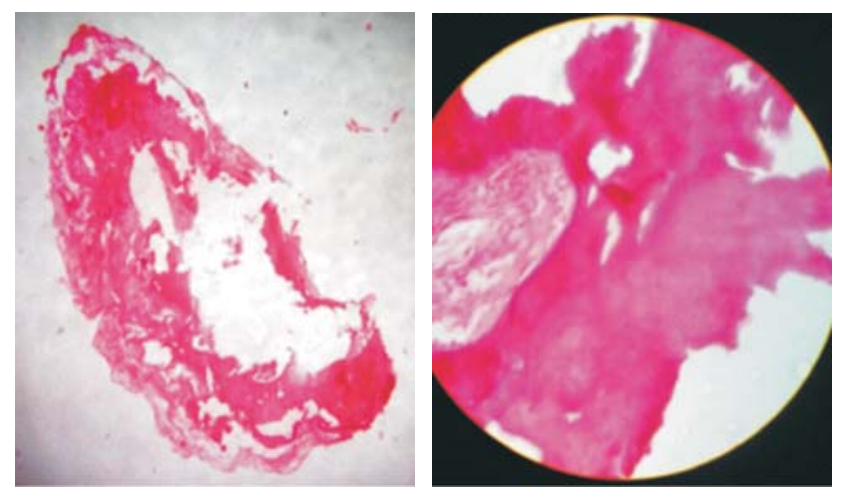

Figure 6: H\&E (4x and 40x) Stained section shows hard tissue interspersed within the connective tissue stroma.

The histopathological report confirms the provisional diagnosis of focal cemento osseous dysplasia. Healing was uneventful.

\section{DISCUSSION}

Focal cemento-osseous dysplasia is first reported as localised fibroosseous lesion by Waldron and later renamed as focal cemento-osseous dysplasia and its features was described by Summerlin and Tomlich. ${ }^{4}$ The aetiology and pathogenesis of FCOD remain unknown, and this lesion is considered to be a reactive or dysplastic process in periapical tissues. ${ }^{5}$ 
Although the lesion is not considered to be odontogenic, it is undoubtedly related to the presence of teeth. Almost all FCODs appear above the mandibular canal and are thus confined to the alveolar process, suggesting at least some odontogenic influence on their genesis. ${ }^{6}$ In our case, there was no history of trauma so the lesion may be developmental in origin.

Focal sclerosing osteomyelitis is characterised by an asymptomatic, non-expansile periapical lesion associated with a tooth exhibiting deep caries. FCODs are usually not infected. FCOD is commonly seen in 3 rd-5th decades in females and mandible is predisposed to these lesions. Our case was in accordance with gender predilection and was seen in same age range and in mandible.

The presence of inflammation is critical for the diagnosis of focal sclerosing osteomyelitis because this lesion can resemble several other intrabony processes that produce somewhat similar histological patterns. ${ }^{8}$ The differential diagnosis should consider the stage of development of the lesion and include periapical granuloma or cyst and chronic osteomyelitis at osteolytic stage, whereas in the mixed and radio opaque stages, like our case, chronic sclerosing osteomyelitis, ossifying/cementifying fibroma, cementoblastoma should be considered. ${ }^{2}$

Radiographically these lesion appears as well defined radiolucent, mixed radiolucent- radioopaque or with time and increasing mineralization, the lesions become progressively radiopaque but rarely exceed 1 $\mathrm{cm}$ in diameter. FCOD is usually radiopaque, as in our case, which exhibited a 2-cm-diameter sclerotic mass with a radiolucent border on panoramic radiography.

Histological examination helps to distinguish focal cemento-osseous dysplasia from cementossifying fibroma. In case of focal cemento osseous dysplasia thick curvilinear bony trabeculae, irregular cementoid masses, loose collagen fibres and free haemorrhage are seen where as in cement-ossifying fibroma there is presence of thin isolated trabeculae with prominent osteoblastic rimming, ovoid cementoid deposits and dense collagen fibres. ${ }^{9}$
Unless focal cemento-osseous dysplasia is symptomatic, treatment is usually not needed but progressive deposition of cementum like calcification leads to maturation in cemento-osseous dysplasia leading to ischemia of affected tissues. This can increase the risk of secondary infection and contribute to delayed osteomyelitis and sequestration which therefore requires surgical intervention.

The management of COD is conservative and follow up is required as it can progress in to florid cemento-osseous dysplasia although, superimposed infection can occur when the lesion becomes exposed into the oral cavity following tooth extraction or erosion of the overlying mucosa. Osteomyelitis resulting from infected lesions is difficult to treat as there is widespread sclerosis with consequent poor blood supply. ${ }^{2}$ However, in our case patient came with chief complaint of pain in region where radiopacity was present so surgical procedure was warranted as postulated in literature.

\section{CONCLUSION}

Plethora of periapical lesions masquerade each other so it is mandatory to do differential diagnosis to avoid mismanagement. Although Focal cementoosseous dysplasia is asymptomatic so usually no treatment is required and follow up is necessary as transformation in to florid form is reported in literature. But in symptomatic cases surgical removal is necessary.

\section{REFERENCES}

1. Waldron CA. Fibro-osseous lesions of the jaws. J Oral Maxillofac Surg 1993;51: 828-835.

2. Drazic R, Minic AJ. Focal cemento-osseous dysplasia in the maxilla mimicking periapical granuloma. Oral Surg Oral Med Oral Pathol 1999;88(1):87-89.

3. Wood NK and Goaz PW. Differential diagnosis of Oral and Maxillofacial lesions. 5th edn. Mosby, Philadelphia; 1997:462-465.

4. Summerlin DJ and Tomich CE. Focal Cemento- osseous dysplasia: A clinopathological study of 221 cases. Oral Surg Oral Med Oral Pathol Oral Radiol Endod 1994; 78(5): 611620.

5. Dagistan S, Tozoglu U, Goregen M. Florid cemento-osseous 
dysplasia: a case report. Med Oral Patol Oral Cir Bucal 2007; 12: E348.

6. MacDonald-Jankowski DS. Focal cemento-osseous dysplasia: a systematic review. Dentomaxillofac Radiol 2008; 37(6):350-360.

7. Melrose RJ . The clinico-pathological spectrum of cementoosseous dysplasia. Oral Maxillofac Clin Nor Am 1997;9:643653.
8. Cankaya AB, Erdem MA, Olgaz V and Firat DR. Focal cemento-osseous dysplasia of mandible.BMJ Case Reports 2012.

9. Su L,Weathers DR and Waldron CA. Distinguishing features of focal cemento-osseous dysplasias and cemento-ossifying fibromas: I.A pathologic spectrum of 316 cases. Oral Surg Oral Med Oral Pathol Oral Radiol Endod 1997; 84(3):301309. 